\title{
Protective Effects of Cerebrospinal Fluid With The Intake of Xixin Decoction on Hippocampal Neuron Apoptosis Induced by $\mathrm{A} \beta_{1-42}$
}

\section{Diwu Yong-chang ${ }^{1^{*}}$, Meng Ting-ting ${ }^{1}$, Wang Fang ${ }^{1}$, Gu Zan ${ }^{1}$, Zeng Jian ${ }^{1}$, Yang Ke ${ }^{1}$, Cui $\mathrm{Na}^{2}$ and Gu Jie ${ }^{3}$}

${ }^{1}$ The First Clinical Medical College, Shaanxi University of Chinese Medicine, People's Republic of China

${ }^{2}$ The College of Foreign Languages, Shaanxi University of Chinese Medicine, People's Republic of China

${ }^{3}$ The Medical Research Center, Shaanxi University of Chinese Medicine, People's Republic of China

"Corresponding author: Diwu Yong-chang, The First Clinical Medical College, Shaanxi University of Chinese Medicine, People's Republic of China, E-mail: diwuyongchang@126.com

Received date: February 10, 2017; Accepted date: March 10, 2017; Published date: March 13, 2017

Copyright: (c) 2017 Yong-chang et al. This is an open-access article distributed under the terms of the Creative Commons Attribution License, which permits unrestricted use, distribution, and reproduction in any medium, provided the original author and source are credited.

\begin{abstract}
Objective: To study the protective effects of cerebrospinal fluid with the intake of Xixin Decoction (CSF) on

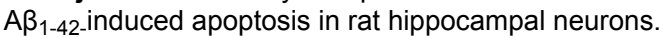

Methods: Hippocampal neurons were extracted from Wistar newborn rats and cultured in vitro. Cultured neurons were divided into the following groups: the normal control group, the $A \beta$ model group, the low concentration treatment group, the medium concentration treatment group, and the high concentration treatment group. We analyzed cell viability using an MTT assay. Cell apoptosis was observed using transmission electron microscopy, and the rate of cell apoptosis was measured with flow cytometry using Annexin V/PI double staining kit. The gene expression and protein expression levels of Caspase-9, Bcl-2, and Bax in cells were measured using Real-time $\mathrm{PCR}$, Western blot, and immunofluorescence techniques.
\end{abstract}

Results: cerebrospinal fluid with the intake of Xixin Decoction was found to improve the survival rate of cells, improve cell morphology during apoptosis, and reduce the number of apoptotic cells. Compared with the control group, the expression levels of Caspse- 9 and Bax were increased in the model group, while the Bcl-2 expression level was decreased. In addition, the expression levels of Caspase- 9 and Bax were found to be reduced in each treatment group, compared with the model group. It was also quite evident that Bcl-2 expression levels were increased $(\mathrm{P}<0.05$ or $\mathrm{P}<0.01)$.

Conclusion: cerebrospinal fluid with the intake of Xixin Decoction exhibits a significant protective effect on cells against $A \beta_{1-42}$-induced apoptosis. Importantly, we demonstrate a dose-effect relationship, with the effects of cerebrospinal fluid with the intake of Xixin Decoction at a density of $20 \%$ showing the most significant results.

Keywords: Alzheimer's disease; A $\beta 1-42$; Xixin decoction; Cell apoptosis

\section{Introduction}

Alzheimer's disease (AD) is a genetic neurodegenerative disease of the central nervous system that has been closely linked with age. $\mathrm{AD}$ is characterized by neuropathology with brain neuritic plaques (Senile plaque, SP) and neurofibrillary tangles (NFTs). Currently, the etiology and pathogenesis of $\mathrm{AD}$ are not yet fully understood. In the field, it is known that $\beta$-amyloid protein $(A \beta)$ deposits are a key pathogenic feature of $\mathrm{AD}$. This initiated the "A $\beta$ cascade theory", which has been widely accepted by scholars. This theory suggests that $\mathrm{AD}$ is caused by an abnormal accumulation of $A \beta$, which can cascade in various forms, exerting toxic effects on mature neurons and ultimately causing the onset of apoptosis of neurons and neuronal degeneration [1].

Traditional Chinese medicine has been used in the treatment of nervous system degenerative diseases such as AD. Traditional Chinese medicine is a beneficial treatment option as it is able to reach multiple targets and produce fewer side effects. Xixin decoction is a prescription drug in the Qing Dynasty, used by Chen Shiduo as a treatment for dementia. There exists 100 years of clinical work representing the use of this drug for the treatment of dementia. In these studies, preliminary experimental work also demonstrated that in the model of AD by intracerebroventricular injection of STZ (streptozocin) Xixin decoction improved the spatial learning and memory capability of rats [2]. Thus, based on previous work, we carried out in vitro experiments to gain insight into the application of Chinese medicine cerebrospinal fluid theory to specifically explore the effect of Xixin Decoction on A $\beta$ induced neuronal apoptosis, as well as the underlying mechanism.

\section{Materials and Methods}

\section{Materials}

1. Laboratory animals: New Zealand rats were obtained from the experimental animal center of The Fourth Military Medical University (license number: SCXK- (Army) 2012-0006). Clean grade Wistar rats were purchased from Beijing Weitong Lihua Experimental Animal Technology Co. Ltd. (license number: SCXK- (Beijing) 2012-0001).

2. Main reagents and drugs: Xixin decoction prescription granules were purchased from Sichuan New Green Pharmaceutical Co. Ltd., 
China (batch number: 1312044). Ginsenoside Rg1 was purchased from Chengdu Mansite Biotechnology Limited Company, China (batch number: 22427-39-0, with a purity greater than 98\%). B27 Neurobasal neurotrophic factor, DMEM/F12 medium, and L-glutamine were purchased from Gibco, USA. Lyophilized $A \beta_{1-42}$ powder and poly-Llysine were purchased from Sigma, USA. $\beta$-tubulin 3 was purchased from Beijing Biosynthesis Company, China. Goat anti-rabbit antibody, and AnnexinV-FITC/PI cell apoptosis detection kit were purchased from Wuhan Boster Company, China. Trypsin was purchased from Beijing Cable Company, China. Inoculum medium, DMEM/F12 (1:1) HEPES solution, $10 \%$ fetal bovine serum, serum-free medium, Neurobasal medium+B27 additives, chloral hydrate, skim milk powder, anti-Bcl-2 antibody [Bcl2/100] (ab117115), anti-Caspase-9 (E23) (antibody (ab32539), and anti-Bax antibody (E63) (ab32503) were purchased from Abcam, USA.

primers TaKaRa co., LTD

RATBCL-2-F 5 '-3' GAGGGGCTACGAGTGGGATA

RATBCL-2-R 5 '-3' CGGTAGCGACGAGAGAAGTC'

RATBax-2-F 5 '-3' GGCGAATTGGCGATGAACTG'

RATBax-2-R 5 '-3' ATGGTTCTGATCAGCTCGGG'

RATcaspase9-F 5 '-3' AGCTGGCCCAGTGTGAATAC'

RATcaspase9-R 5 '-3' GCTCCCACCTCAGTCAACTC'

\section{Methods}

1. Oligomeric $A \beta_{1-42}$ preparation: Freeze-dried $A \beta_{1-42}$ powder was dissolved in saline solution to a final concentration of $100 \mu \mathrm{mol} \cdot \mathrm{L}^{-1}$, incubated at $37^{\circ} \mathrm{C}$ for $7 \mathrm{~d}$. Before use, oligomeric $\mathrm{A} \beta_{1-42}$ was diluted to the desired concentration in serum-free medium according to the experimental protocol.

2. Preparation and HPLC detection of cerebrospinal fluid with the intake of Xixin Decoction: Male New Zealand rats with a body weight $(2+0.3) \mathrm{kg}$ were used for these experiments. Xixin decoction prescription granules were administered by gavage. Daily oral doses for human and rabbit body surface areas were calculated using the described formula. Gavage was performed once a day for $5 \mathrm{~d}$. At the end of the last gavage, cisternal cerebrospinal fluid was extracted via percutaneous puncture. Taking ginsenoside $\operatorname{Rg} 1$ as index ingredients, cerebrospinal fluid with the intake of Xixin Decoction and ginsenoside Rg1 control solution after pretreatment are detected with HPLC.

3. Primary rat hippocampal neuronal culture: Hippocampi was dissected from Wistar rat within $24 \mathrm{~h}$ following birth. The meninges were removed and cut into pieces, incubated with $0.125 \%$ trypsin at $37^{\circ} \mathrm{C}$ for $5 \mathrm{~min}$. They were then gently passed through a $200-\mathrm{mesh}$ sieve to obtain a single cell suspension. Cells were then counted and plated in either 6- or 96-well culture plates. Following $24 \mathrm{~h}$, the media were removed and cells were cultured in serum-free medium, which was replaced once every two days. Cell growth was observed using an inverted microscope. After 5-7 d, if the cells were in good condition, they were harvested for the experiments.

4. Cell groups and treatments: Cultured cells were divided into the following 5 groups: 1) Blank control group: normal cultured cortical neurons; 2) Model group: containing $25 \mu \mathrm{mol} \cdot \mathrm{L}^{-1}$ oligomeric $\mathrm{A} \beta_{1-42}$ in serum-free medium; 3$)$ the low concentration treatment group $(5 \%$ $+\mathrm{A} \beta$ group): cultured in serum-free medium containing $25 \mu \mathrm{mol} \cdot \mathrm{L}^{-1}$ oligomeric $A \beta_{1-42}$ and $5 \%$ cerebrospinal fluid with the intake of Xixin
Decoction; 4) the medium concentration treatment group $(10 \%+\mathrm{A} \beta$ group): cultured in serum-free medium containing $25 \mu \mathrm{mol} \cdot \mathrm{L}^{-1}$ oligomeric $A \beta_{1-42}$ and $10 \%$ cerebrospinal fluid with the intake of Xixin Decoction; 5) the high concentration treatment group $(20 \%+A \beta$ group): containing $25 \mu \mathrm{mol} \cdot \mathrm{L}^{-1}$ oligomeric $A \beta_{1-42}$ and $20 \%$ cerebrospinal fluid with the intake of Xixin Decoction.

5. MTT assay: administration in accordance with the "cell groups and treatments". Hippocampal neurons were treated for $24 \mathrm{~h}, 48 \mathrm{~h}$ and $72 \mathrm{~h}$, and then MTT solution $\left(5 \mathrm{mgmL}^{-1}\right)$ was added to each well $(20$ $\mu \mathrm{l} /$ well) and cultured for $4 \mathrm{~h}$, after which the supernatant was removed and $150 \mu \mathrm{L}$ DMSO was added to each well. The absorbance (A) values were measured at $570 \mathrm{~nm}$ using a plate reader. The survival rate of cells was calculated according to the following formula: cell survival rate $=\mathrm{A}$ (experimental group)/A (control group) $\times 100 \%$. Each group has 4 parallel samples and each experiment was repeated for 3 times.

6. Transmission electron microscope analysis: Sample preparation: cells were digested using $0.125 \%$ trypsin, harvested and washed twice with PBS, followed by fixation with $4 \%$ glutaraldehyde. Samples were then prepared as described previously (reference), and imaged using electron microscopy.

7. Annexin V/PI double staining assay: Cells were treated for $24 \mathrm{~h}$, $48 \mathrm{~h}$ or $72 \mathrm{~h}$, harvested and washed twice with PBS. Cells were then stained with Annexin V-FITC kit by following the instructions of manufacture, and analyzed by flow cytometry.

8. Real-time PCR: Hippocampal neurons were treated for $24 \mathrm{~h}, 48 \mathrm{~h}$ or $72 \mathrm{~h}$. Each group of cells was mixed with $1 \mathrm{ml}$ Trizol and brought to a uniform homogenate. RNA extraction was then carried out. RNA identification was done to ensure RNA integrity and to determine the concentration. The complete reverse transcription reaction was performed in a $20 \mu \mathrm{l}$ reaction volume. The positive standard and testing samples were prepared at the same time on the machine, using the following reaction conditions: $93^{\circ} \mathrm{C}$ for $2 \mathrm{~min}, 93^{\circ} \mathrm{C}$ for $1 \mathrm{~min}$, and $55^{\circ} \mathrm{C}$ for $2 \mathrm{~min}$, for a total of 40 cycles. mRNA levels were detected by Real-time PCR. Using GAPDH as the reference gene, the Ct value was used for relative quantitative analysis, the relative expression of $2-\mathrm{Ct}$ as RNA.

9. Western blot: Hippocampal neurons were treated for $24 \mathrm{~h}, 48 \mathrm{~h}$ or $72 \mathrm{~h}$. Cells were then washed with PBS and lysed using an ultrasonic treatment. Lysate was then centrifuged, and the cleared lysate was loaded onto a PAGE gel. After running the gel, samples were transferred to a PVDF membrane and blocked with 5\% FBS, and then the membrane was incubated with primary antibody at $4^{\circ} \mathrm{C}$ overnight. The membrane was then washed 3 times in TBST and incubated with secondary antibody at room temperature for 1-2 h. Next, the light emitting A solution and B solution were mixed and added uniformly to the membrane. The membrane was then exposed to film.

10. Immunofluorescence assay: Hippocampal neurons on coverslips were treated for $24 \mathrm{~h}, 48 \mathrm{~h}$ or $72 \mathrm{~h}$. Cells were fixed in $4 \%$ paraformaldehyde, washed, incubated for $20 \mathrm{~min}$ in $0.3 \%$ Triton X-100 at room temperature, washed, and then incubated in non-immune serum at room temperature for $10 \mathrm{~min}$. Coverslips were then incubated with primary antibody at $4^{\circ} \mathrm{C}$ overnight, washed and incubated with secondary antibody conjugated to fluorescein at $37^{\circ} \mathrm{C}$ for $30 \mathrm{~min}$ in the dark. Coverslips were then washed and incubated with DAPI for $1520 \mathrm{~min}$ to stain nuclei. Coverslips were finally mounted in glycerol phosphate buffer solution for observation using fluorescence microscopy. Acquired images were then analyzed. 
Citation: Yong-chang D, Ting-ting M, Fang W, Zan G, Jian Z, et al. (2017) Protective Effects of Cerebrospinal Fluid With The Intake of Xixin Decoction on Hippocampal Neuron Apoptosis Induced by A $\beta_{1-42}$. J Aging Sci 5: 175. doi:10.4172/2329-8847.1000175

Page 3 of 6

\section{Statistics}

The experimental data were analyzed by statistical software SPSS 19 , and shown as mean $\pm \mathrm{SD}$. Each group was compared using the t test, and multigroup mean single factor analysis of variance (F test). Multiple comparison between groups was performed using LSD-t test, and $\mathrm{P}<0.05$ was considered statistically significant.

\section{Results}

\section{HPLC detection of cerebrospinal fluid active ingredients of ginsenoside Rg1}

The HPLC results demonstrate that comparing the corresponding positions of the $\operatorname{Rg} 1$ control sample and the sample solution chromatogram from each group, the same retention time (about 77.8 min) of the chromatographic peak was observed, with the quality control sample exhibiting no interference (Figure 1).

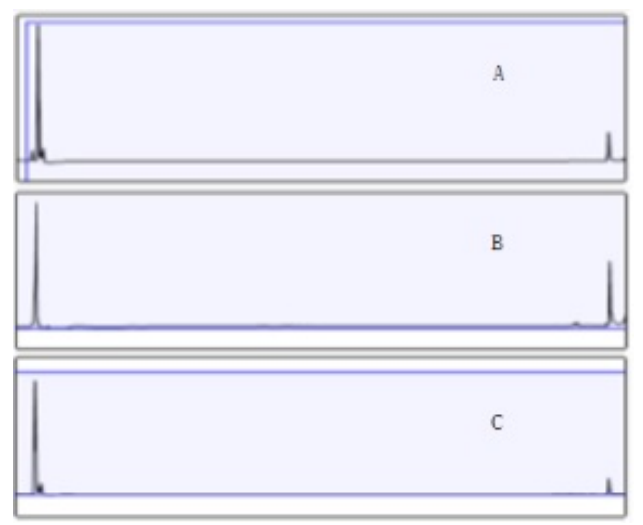

Figure 1: Chromatographic peak was observed, with the quality control sample exhibiting no interference.

\section{Effect of cerebrospinal fluid with the intake of Xixin Decoction on cell survival}

Compared with the blank group, the cell number in the model group was found to be significantly decreased $(\mathrm{P}<0.01)$. After $48 \mathrm{~h}$ treatment with $25 \mu \mathrm{mol} \cdot \mathrm{L}^{-1} \mathrm{~A} \beta_{1-42}$, the relative cell survival rate of the model group was $(86.37+9.56) \%$. Incontrast, after treatment with $5 \%$, $10 \%$ and $20 \%$ cerebrospinal fluid with the intake of Xixin Decoction , respectively, the cell survival rate was significantly increased dosedependently by $20.61 \pm 11.93 \%$ and $47.10 \pm 2.13 \%$, respectively compared with the model group $(\mathrm{P}<0.05$ or 0.01$)$, (Figure 2$)$.

\section{Effect of cerebrospinal fluid with the intake of Xixin Decoction on neuronal apoptosis}

1. Observation of morphology by transmission electron microscope: The transmission electron microscope analysis showed that the number of cells in the model group decreased compared to the control group. In addition, cells were found to be decreased in size, round up, lose cell membrane microvilli, possess an irregular nuclear morphology, possess nucleolus margination, and nuclear chromatin was observed to be of a "black hole" shape and have an uneven distribution. In addition, the cytoplasm exhibited a vacuolar structure,

possessed a lot of granular material, had a decreased number of mitochondria, and had an increased number of lysosomes.

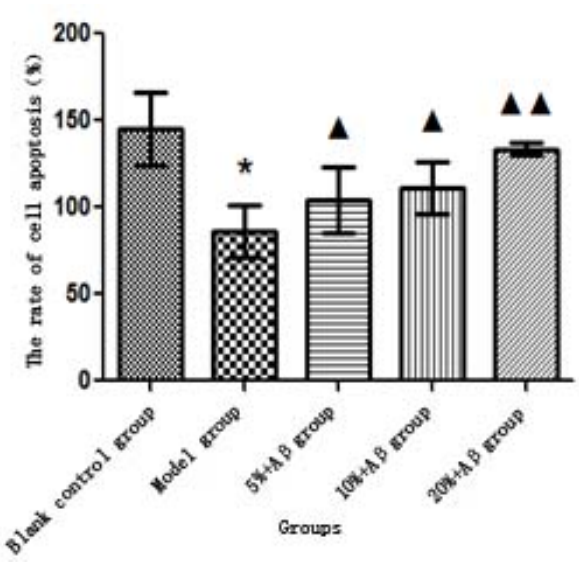

Figure 2: Comparison between the rate of cell apoptosis (\%) and model groups.

However, the cell membrane was found to remain intact. Interestingly, fewer apoptotic cells and no significant changes in apoptosis morphology were observed in the treatment group compared with the model group (Figure 3).

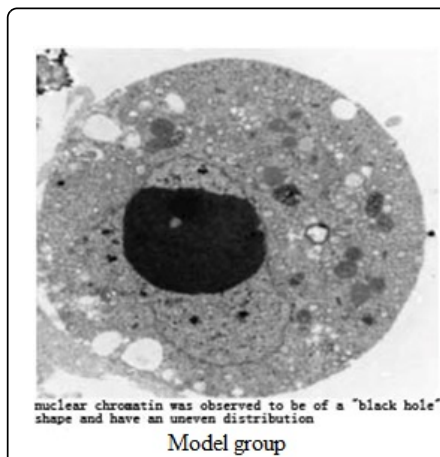

Figure 3: Fewer apoptotic cells and no significant changes in apoptosis morphology were observed in the treatment group compared with the model group.

2. Cell apoptosis analyzed using flow cytometry: The rate of apoptosis was found to be lower in the blank control group compared to the model group. Compared with the blank control group, the apoptosis rate in the model group increased to $29.2 \%-55.2 \%$. In contrast, the apoptosis rate in the treatment groups (5\%,10\% and $20 \%$ of Xixin decoction) gradually decreased to $19.3 \%$, compared with the model group. This suggests that cerebrospinal fluid with the intake of Xixin Decoction can significantly protect cells and reduce the number of apoptotic cells (Figures 4 and 5).

3. Real-time PCR analysis results: (As shown in Table 1 and Figure 6) Compared with the blank group, the Caspase- 9 mRNA and Bax mRNA levels in the model group were increased $(\mathrm{P}<0.05$ or $\mathrm{P}<0.01)$, while those of the treatment groups $(48 \mathrm{~h})$ were significantly decreased compared with the model group $(\mathrm{P}<0.05$ or $\mathrm{P}<0.01)$. 
Citation: Yong-chang D, Ting-ting M, Fang W, Zan G, Jian Z, et al. (2017) Protective Effects of Cerebrospinal Fluid With The Intake of Xixin Decoction on Hippocampal Neuron Apoptosis Induced by A $\beta_{1-42}$. J Aging Sci 5: 175. doi:10.4172/2329-8847.1000175

Page 4 of 6

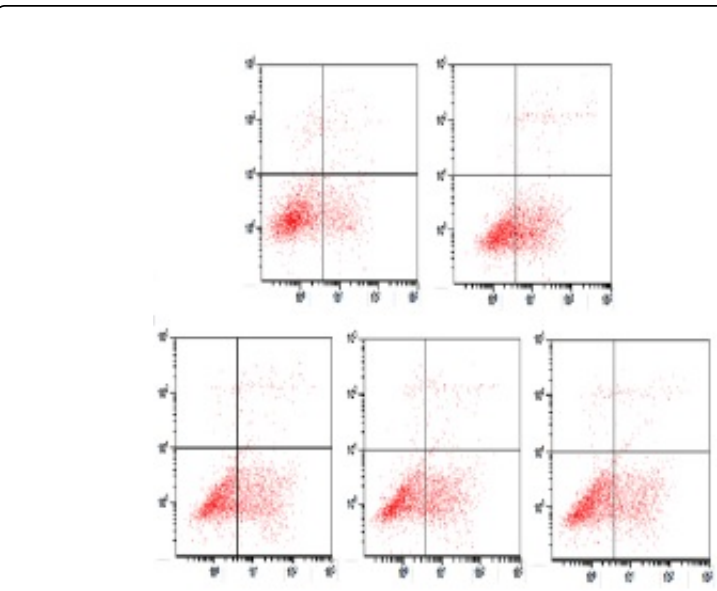

Figure 4: Cerebrospinal fluid with the intake of Xixin Decoction can significantly protect cells and reduce the number of apoptotic cells.

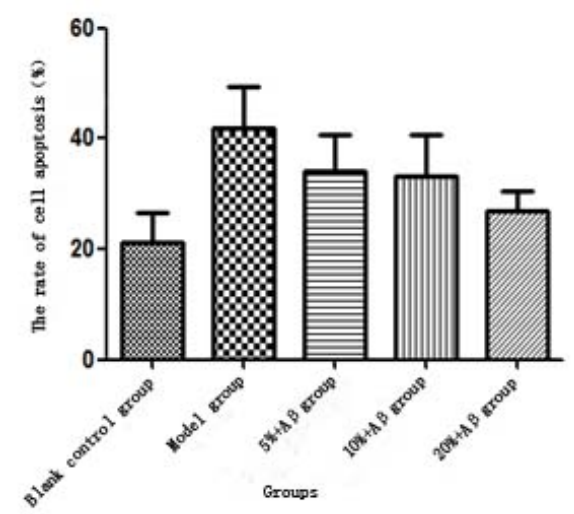

Figure 5: Comparison between the apoptosis rate and the model group.
Compared with blank group, the expression of Bcl-2 mRNA in the model group was reduced $(\mathrm{P}<0.05$ or $\mathrm{P}<0.01)$, while those of the treatment groups $(48 \mathrm{~h})$ were significantly increased compared with the model group $(\mathrm{P}<0.05$ or $\mathrm{P}<0.01)$. These data demonstrate that $\mathrm{Xi}$ Xin Decoction with CSF can inhibit the gene expression of factors involved in triggering apoptosis, and increase the gene expression of anti-apoptotic factors, all leading to the inhibition of apoptosis. The most pronounced effect was observed in the highest concentration treatment group.

\begin{tabular}{|l|l|l|l|}
\hline Group & Caspase-9 & Bax & Bcl-2 \\
\hline Blank group & $1.0 \pm 0.17$ & $1.0 \pm 0.05$ & $1.0 \pm 0.01$ \\
\hline Model group & $1.5 \pm 0.26$ & $1.2 \pm 0.03$ & $0.9 \pm 0.02$ \\
\hline $\begin{array}{l}\mathrm{A} \beta+5 \% \mathrm{CSF} \\
\text { group }\end{array}$ & $0.2 \pm 0.07 \mathbf{\Lambda}$ & $1.1 \pm 0.07 \mathbf{\Lambda}$ & $1.0 \pm 0.13^{\mathbf{\Lambda}}$ \\
\hline $\begin{array}{l}\mathrm{A} \beta+10 \% \mathrm{CSF} \\
\text { group }\end{array}$ & $0.1 \pm 0.25^{\mathbf{\Lambda}}$ & $1.1 \pm 0.09 \mathbf{\Lambda}$ & $1.0 \pm 0.02 \mathbf{\Lambda}$ \\
\hline $\begin{array}{l}\mathrm{A} \beta+20 \% \mathrm{CSF} \\
\text { group }\end{array}$ & $0.1 \pm 0.12 \mathbf{\Lambda}$ & $0.8 \pm 0.07 \mathbf{\Lambda}$ & $1.3 \pm 0.06 \mathbf{\Lambda}$ \\
\hline $\begin{array}{l}\text { Note: compared } \\
\text { P0.05,P0.01 }\end{array}$ & with blank group: P0.05P0.01compared with model group: \\
\hline
\end{tabular}

Table 1: Real-time PCR analysis results.

4. Western blot analysis results: (As shown in Figure 7) Compared with the blank group, the protein expression levels of Caspase- 9 and Bax were found to be increased in the model group, while the expression levels of these two proteins in the treatment groups were found to be reduced compared to the model group. In contrast, the Bcl-2 protein expression level in the model group was reduced compared with the blank group, while the Bcl-2 protein expression levels in the treatment groups were significantly increased compared with model group.
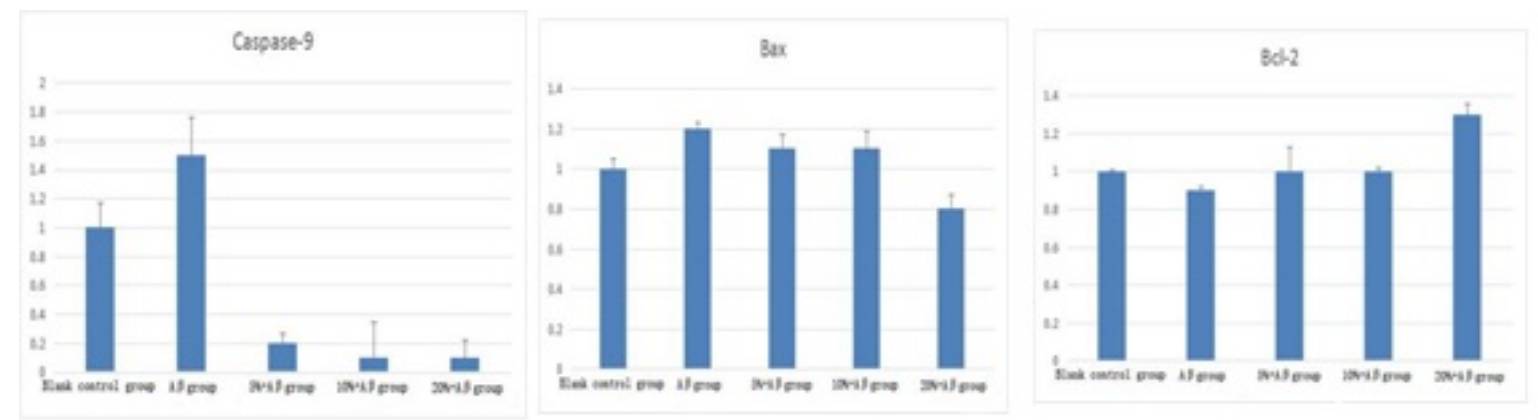

Figure 6: compared with the blank group.

5. Immune fluorescence testing results: The immune fluorescence testing results showed that the Caspase- 9 and Bax protein levels were increased in the model group compared to the blank group, while these levels were much lower in the treatment groups compared to the model group. There was a clear reduction in cell number in the model group compared to the blank group. We found that Bcl-2 protein level was reduced in the model group compared to the blank group, while the Bcl-2 protein levels in the treatment groups were significantly increased compared to the model group (Figures 8-10). 
Citation: Yong-chang D, Ting-ting M, Fang W, Zan G, Jian Z, et al. (2017) Protective Effects of Cerebrospinal Fluid With The Intake of Xixin Decoction on Hippocampal Neuron Apoptosis Induced by A $\beta_{1-42}$. J Aging Sci 5: 175. doi:10.4172/2329-8847.1000175

Page 5 of 6

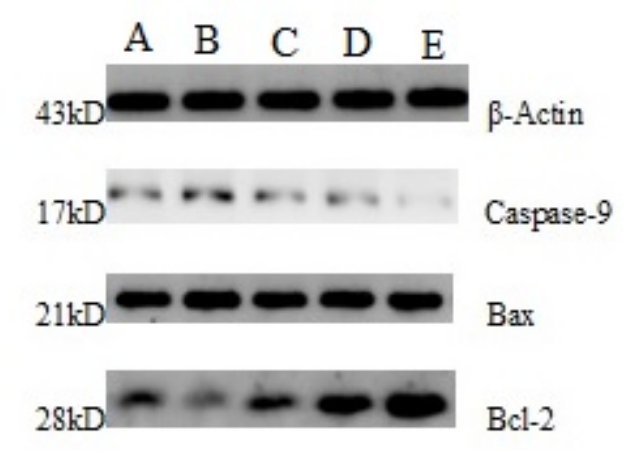

Figure 7: Western blot analysis results.
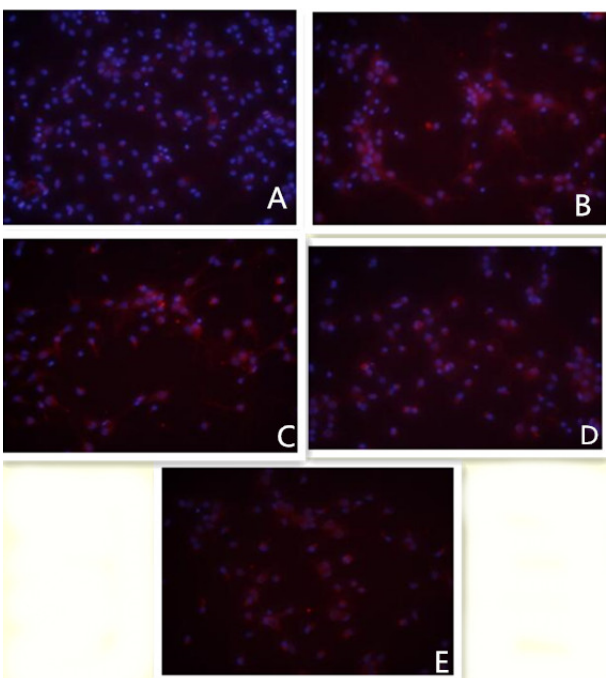

Figure 8: Caspase-9 A=blank group; $\mathrm{B}=$ model group; $\mathrm{C}, \mathrm{D}$ and $\mathrm{E}$, low/middle/high concentration groups.

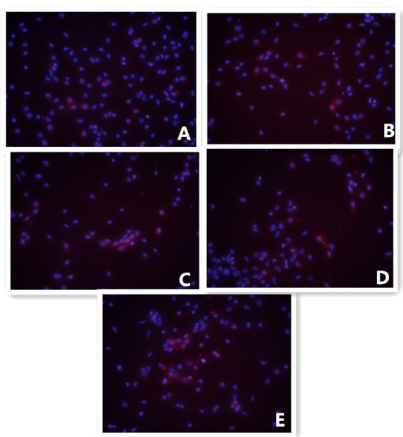

Figure 9: $\mathrm{Bax} \mathrm{A}=$ blank group; $\mathrm{B}=$ model group; $\mathrm{C}, \mathrm{D}$ and $\mathrm{E}$, low/ middle/high concentration groups.

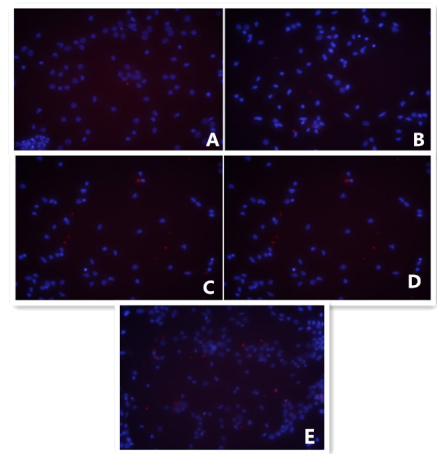

Figure 10: $\mathrm{Bcl}-2 \mathrm{~A}=$ blank group; $\mathrm{B}=$ model group; $\mathrm{C}, \mathrm{D}$ and $\mathrm{E}$, low/ middle/high concentration groups.

\section{Discussion}

Apoptosis, also referred to as programmed cell death (PCD), is a cell suicide type of process that is triggered by signals generated by various stimuli within the body. Until now, it has been thought that $A \beta$ is the key pathogenic factor involved in $\mathrm{AD}$. This has been widely agreed upon by numerous scholars, resulting in the generation of the "A $\beta$ cascade theory", which has become one of the most common theories used to explain the pathogenesis of AD. Studies have confirmed that over-generation of $A \beta$ or a metabolic disturbance can lead to abnormal $\mathrm{A} \beta$ accumulation both in the hippocampal and cortical areas of the AD brain. This is thought to be the primary reason behind neuronal injury or even apoptosis and synaptic loss in those areas. Neuronal apoptosis and synaptic loss are two pathological features of $\mathrm{AD}$, and a potential cause of $\mathrm{AD}$ as well. Early stage memory loss in $\mathrm{AD}$ patients is caused by the destruction of synaptic plasticity. In advanced $A D$, severe dementia is caused by large amounts of neuronal degeneration and apoptosis, both of which are closely tied to A $\beta$ 's neurotoxicity. Studies have shown that soluble $A \beta$ oligomers carry greater neurotoxicity and can lead to synaptic damage in early stage $\mathrm{AD}$, and cause persistent synaptic plasticity. It is thought to be more powerful in destructing the physiology of learning and memory. Thus, it is thought that soluble A $\beta$ oligomers could be the "initiation factor" of $\mathrm{AD}$ in the early stages of AD pathology [3-5].

$\mathrm{A} \beta$ ' s neurotoxicity causes apoptosis of neurons mainly through a cascading effect of caspases [6]. A $\beta$ induces an apoptotic mechanism, affecting the expression of apoptosis genes, and the Bcl-2 family proteins play a critical role in this apoptotic signal transduction in cells. The Bcl-2 family includes apoptotic inhibitory factors such as $\mathrm{Bcl}-2$ and $\mathrm{Bcl}-\mathrm{xL}$, and apoptogenic factors including Bax and Bak. The balance of expression levels of Bcl-2 and Bax plays an important role in the apoptosis route [7]. Because $\mathrm{Bc1}-2$ is a stable protein and can play a role in blocking cell death, it can inhibit apoptosis by changing the concentration of $\mathrm{Ca}^{2+}$ in organelles within the cell [8]. Bax has the opposite effect, where the generation of Bax homodimers induces apoptosis.

The Chinese medicine "Compound Xixin Decoction" that was used in this study is a popular ancient prescription that was used for the treatment of $\mathrm{AD}$ patients. The long period of clinical validation proved that this prescription generates a positive effect on $\mathrm{AD}$ patients. This 
Citation: Yong-chang D, Ting-ting M, Fang W, Zan G, Jian Z, et al. (2017) Protective Effects of Cerebrospinal Fluid With The Intake of Xixin Decoction on Hippocampal Neuron Apoptosis Induced by A $\beta_{1-42}$. J Aging Sci 5: 175. doi:10.4172/2329-8847.1000175

Page 6 of 6

medicine consists of ginseng, calamus, pinellia, poria cocos, jujube, and others. Old pharmacological research suggests that ginseng and shi changpu possess anti-apoptotic effects. It is previously shown that both ginsenoside $\mathrm{Rgl}$ and estrogen can alleviate the toxic effect on neurons, and result in an increase in anti-apoptotic factors and protein expression levels, reducing the apoptogenic factor Bax mRNA and protein expression [9]. Acorus gramineus Soland's important component $\beta$-asarone exhibits a clear protective function to prevent apoptosis in cortical neurons induced by $\mathrm{AlCl}_{3}$ in mice, which is able to block intracellular $\mathrm{Ca}^{2+}$ accumulation and reduce apoptosis in neurons [10].

In this study, we tested the Xixin Decoction's main component ginsenoside Rg1 in animal cerebrospinal fluid using HPLC. We found that the compared sample and the sample solution in each group possessed the same relative position of their chromatographic peak on the chromatogram, with the same retention time of approximately 77.8 min, with the samples not showing interference. This suggests that the effective components of the Chinese compound medicine are able to penetrate the blood-brain barrier and work on neurons within the brain. This study demonstrates that the oligomeric $A \beta_{1-42}$ clearly reduced the survival rate of cells and increased apoptosis. During this study, we found that cerebrospinal fluid with the intake of Xixin Decoction was able to improve viability of hippocampal neurons, and reduce the apoptosis rate. At the same time, cerebrospinal fluid with the intake of Xixin Decoction was also found to inhibit Caspase-9 and Bax protein expression, increase $\mathrm{Bcl}-2$ protein expression, suggesting its obvious protective effect to prevent cell apoptosis induced by $A \beta_{1-42}$, with a quantity-effect relationship observed. cerebrospinal fluid with the intake of Xixin Decoction with a $20 \%$ concentration was shown to exhibit the best effect. It should be noted that these experimental results are consistent with those of previous studies.

In summary, Xi Xin Decoction exhibits an obvious protective effect to prevent apoptosis induced by $\mathrm{A} \beta$ toxicity. Thus, this prescription has a potential application value in the prevention and treatment of $\mathrm{AD}$ patients. However, the mechanism of action of the Chinese compound medicine Xi Xin Decoction's in its prevention and treatment of $\mathrm{AD}$ needs further study.

\section{Author Contributions}

Diwu Yong-chang is the project leader, who is responsible for the project design, establishment of the experimental scheme and the instruction of experimental processes. Cui $\mathrm{Na}$ carried out the Statistical Data Analysis, and revised and polished the paper. Gu Jie is committed to cell culture, molecular-biological indicator test and image analysis. Meng Ting-ting, Zeng Jian, Wang Fang, Gu Zan and Yang Ke are postgraduates studying in university, who are responsible for cell isolation and culture, cell morphological observation, cellular and molecular biological detection.

\section{Conflict of Interest}

No interest conflict.

\section{Funding}

This study is funded by the National Natural Science Foundation of China (No.81373703; No.81674042).

\section{References}

1. Yankner BA, Duffy LK, Kirschner DA (1990) Neurotrophic and neurotoxic effect of Amyloid $\beta$-protein: reversal by tachykinin Neuropeptides. Science 250: 279-282.

2. Yong-chang Diwu, Jin-zhou Tian, Jing Shi (2011) The Effect of Chinese herbal medicine Yinsiwei compound on spatial learning and memory ability and the ultrastructure of hippocampal neurons in a rat model of sporadic Alzheimer disease. J Integr Med 9: 209-215.

3. Birnbaum JH, Bali J, Rajendran L, Nitsch RM, Tackenberg C (2015) Calcium flux-independent NMDA receptor activity is required for Abeta oligomer-induced synaptic loss. Cell Death Dis 6: e1791.

4. Huang HC, Chang P, Lu S Y, Zheng BW, Jiang ZF (2015) Protection of curcumin against amyloid-beta-induced cell damage and death involves the prevention from NMDA receptor-mediated intracellular $\mathrm{Ca}(2+)$ elevation. J Recept Signal Transduct Res 35: 450-457.

5. Adzovic L, Domenici L (2014) Insulin induces phosphorylation of the AMPA receptor subunit GluR1, reversed by ZIP, and over-expression of Protein Kinase M zeta, reversed by amyloid beta. J Neurochem 131: 582-587.

6. Vassar R (2007) Caspase-3 cleavage of GGA3 stabilizes BACE: implications for Alzheimer's disease. Neuron 54: 671-673.

7. Zhao ZQ, Nakamura M, Wang NP, Wilcox JN, Shearer S, et al. (2000) Reperfusion induces myocardial apoptotic cell death. Cardiovasc Res 45: 651-660

8. Holst CM, Johansson VM, Alm K, Oredsson SM (2008) Novel antiapoptotic effect of Bcl-2: prevention of polyamine depletion-induced cell death. Cell Biol Int 32: 66-74.

9. Gong Li (2010) Effect and mechanism of ginsenoside Rg1 on the cellular model of Blzheimer's disease in postmenopausal women. Doctoral dissertation of Shandong University.

10. Jiang Li, He Yuping, Zou Yanyan (2007) Effect of asarone on calcium ion concentration in cortical neurons in dementia mice. J Rehabil Med 22: 490-491. 